\title{
Proceedings of the 2015 ISNR Conference: Poster Presentations
}

\author{
Selected Abstracts of Conference Presentations at the 2015 International Society for Neurofeedback \\ and Research (ISNR) 23rd Conference, Denver, Colorado, USA
}

Citation: International Society for Neurofeedback and Research. (2015). Proceedings of the 2015 ISNR Conference: Poster Presentations NeuroRegulation, 2(4), 188-196. http://dx.doi.org/10.15540/nr.2.4.188

Copyright: ( 2015. ISNR. This is an Open Access article distributed under the terms of the Creative Commons Attribution License (CC-BY).

\section{Daytime Cortical Arousal Among Patients with Comorbid Insomnia and Major Depressive Disorder \\ I-Mei Lin, PhD, Che-Cheng Chu, MS, Z. C. Tang, PhD, and Yu-Che Tsai, PhD \\ Kaohsiung Medical University, Kaohsiung, Taiwan}

Background: Insomnia is common in patients with major depressive disorder (MDD); they complain of falling asleep and awakening in the daytime. One of the neuropsychopathological mechanisms of insomnia is cortical hyperarousal. Previous studies explored cortical activities at pre-sleep and sleep stages among patients with primary insomnia and MDD, as well as the physiological arousal in the daytime among patients with chronic insomnia. There were few studies that explored clearly the psychopathological mechanism among patients comorbid with MDD and insomnia. The purpose of this study was to explore the daytime cortical arousal among patients comorbid with MDD and insomnia.

Methods: Fifty patients comorbid with MDD and insomnia were the MDD group that was compared with 35 healthy controls in beta power of electroencephalogram (EEG). All of the participants filled out the Pittsburgh Sleep Quality Index (PSQI). The resting EEG with eye-closed were measured for $5 \mathrm{~min}$ via 19-channel BrainMaster equipment (BrainMaster Technologies, Inc., Bedford, Ohio), where the spectrum analyses showed the beta power (13-32 Hz; as a cortical hyperarousal index) at $\mathrm{Fz}$ and $\mathrm{Cz}$.

Results: This study found that:

(1) After controlling the medication effects, there were positive correlations between PSQI total score, the habitual sleep efficiency, use of sleep medications, and beta power at $\mathrm{Fz}$ and $\mathrm{Cz}$.
(2) There were higher absolute and relative beta power at $\mathrm{Fz}$ and $\mathrm{Cz}$ in the MDD group than that of in healthy controls.

(3) After controlling the medication effect, the MDD group with high sleep disturbance had higher relative beta power at $\mathrm{Fz}$ and $\mathrm{Cz}$ than that of the MDD group with low sleep disturbance.

Conclusion: This study indicated that poor sleep quality was associated with daytime cortical arousal. Patients with MDD had higher cortical arousal at the frontal and central areas than that of healthy controls. These phenomena may reflect the daytime cortical hyperarousal that was found among patients comorbid with MDD and insomnia.

\section{References}

Bonnet, M. H., \& Arand, D. L. (2010). Hyperarousal and insomnia: state of the science. Sleep Medicine Reviews, 14(1), 9-15. http://dx.doi.org/10.1016/j.smrv.2009.05.002

Knott, V., Mahoney, C., Kennedy, S., \& Evans, K. (2001). EEG power, frequency, asymmetry and coherence in male depression. Psychiatry Research: Neuroimaging, 106(2), 123-140. http://dx.doi.org/10.1016/S0925-4927(00)00080-9

Nofzinger, E. A., Buysse, D. J., Germain, A., Price, J. C., Miewald, J. M., \& Kupfer, D. J. (2004). Functional neuroimaging evidence for hyperarousal in insomnia. The American Journal of Psychiatry, 161(11), 2126-2128. http://dx.doi.org/10.1176/appi.ajp.161.11.2126

Perlis, M. L., Merica, H., Smith, M. T., \& Giles, D. E. (2001). Beta EEG activity and insomnia. Sleep Medicine Reviews, 5(5), 365-376. http://dx.doi.org/10.1053/smrv.2001.0151

Sunderajan, P., Gaynes, B. N., Wisniewski, S. R., Miyahara, S., Fava, M., Akingbala, F., ... Trivedi, M. H. (2010). Insomnia in patients with depression: a STAR*D report. CNS Spectrums, 15(6), 394-404. 
Neuromodulation Therapy Based on an

Integration of Prefrontal rTMS and

Neurofeedback for the Treatment of Autism

Estate (Tato) Sokhadze, PhD, Ayman S. El-Baz, PhD,

Allan Tasman, MD, Yao Wang, MS, and Manuel F.

Casanova, $M D$

University of Louisville, Louisville, Kentucky, USA

Background: The study is based on an underlying neuropathological model of autism, which emphasizes "minicolumnar" pathology (Casanova, 2007; Casanova et al., 2006) and cortical lateral inhibition deficits resulting in behavioral abnormalities and executive dysfunctions. We propose that neuromodulation based on lowfrequency repetitive TMS over prefrontal area will enhance lateral inhibition through activation of inhibitory double bouquet interneurons and will be accompanied by EEG alteration that can be operantly conditioned using neurofeedback (NFB) immediately after rTMS session. In prior studies in children with ASD we demonstrated post-TMS improvements in executive functions (Casanova et al., 2012; Sokhadze, El-Baz, Sears, Opris, \& Casanova, 2014), as well as positive effects of prefrontal neurofeedback in ASD (Wang et al., 2014). In the current study each rTMS session was followed by NFB training that we predicted to result in a synergetic response.

Objectives: The overall aim of the study was to investigate behavioral responses, ERP indices of information processing, and coherence of evoked and induced gamma oscillations in children with ASD enrolled either in 18 weekly sessions of combined rTMS-NFB training group or in the wait-list group. The goal of our study was to investigate whether error rate, accuracy, frontal and parietal ERPs and gamma coherence, and clinical behavioral evaluation outcomes will show positive changes in the treatment group as compared to wait-list group.

Methods: We used 18 weekly sessions of $0.5 \mathrm{~Hz}$ rTMS bilaterally over prefrontal cortex (around F3-F5 and F4-F6), $90 \%$ of motor threshold, 200 pulses, followed by prefrontal (FPz) neurofeedback in 20 children with ASD (14.9 years). Another group of children with ASD ( $N=22,15.6$ years) was tested twice within 4 months. Baseline and post-treatment (TMS-NFB or wait) assessments used selective attention tests with EEG/ERP recording and clinical behavioral evaluations (ABC; Aman \& Singh, 1994; and RBS-R; Bodfish, Symons, \& Lewis, 1999).
Results: Post-TMS-NFB evaluations showed decreased irritability and hyperactivity on $A B C$, and decreased total repetitive behaviors scores on RBS. Between group difference in error rate was significant, with the TMS-NFB group showing decrease of error rate $(F=5.62, p=.02)$. Magnitude of the frontal N100 decreased, while amplitude of the P200 to target stimuli increased post-TMS-NFB, demonstrating more effective recognition of targets $(F=5.25, p=.027)$. Similar effects were expressed as well in the parietal P3b. The treatment group showed increase coherence of induced gamma to targets between frontal and temporal sites (F3-T7, $F=6.67, p=.14$ ). Neurofeedback sessions resulted in linear regression of the theta/ratio and increase of gamma power over 18 sessions of treatment.

Conclusions: Improved clinical behavioral evaluation outcomes along with behavioral (RT, accuracy) and functional EEG/ERP measures postTMS-NFB treatment are indicative of more efficient processing information post-treatment. The study represents a pilot translational clinical research exploration where rTMS and neurofeedback were combined, and treatment effects were compared with a wait-list group using clinical, behavioral, and cognitive outcome measures. Preliminary results are very encouraging and warrant further, more rigorous, controlled clinical trials to assess efficacy of proposed integrated neuromodulatory intervention for autism treatment.

\section{References}

Aman, M. G., \& Singh, N. N. (1994). Aberrant Behavior Checklist-Community (Supplementary Manual). East Aurora, NY: Slosson Educational Publications.

Bodfish, J. W., Symons, F. J., \& Lewis, M. H. (1999). Repetitive Behavior Scale. Morganton, NC: Western Carolina Center Research Reports.

Casanova, M. F. (2007). The neuropathology of autism. Brain Pathology, 17(4), 422-433. http://dx.doi.org/10.1111/j.17503639.2007.00100.x

Casanova, M. F., Baruth, J. M., El-Baz, A., Tasman, A., Sears, L., \& Sokhadze, E. (2012). Repetitive transcranial magnetic stimulation (rTMS) modulates event-related potential (ERP) indices of attention in autism. Translational Neuroscience, 3(2), 170-180. http://dx.doi.org/10.2478/s13380-012-0022-0

Casanova, M. F., van Kooten, I., Switala, A. E., van Engeland, H., Heinsen, H., Steinbusch, H. W. M., ... Schmitz, C. (2006). Abnormalities of cortical minicolumnar organization in the prefrontal lobes of autistic patients. Clinical Neuroscience Research, 6(3-4), 127-133. http://dx.doi.org/10.1016 /j.cnr.2006.06.003

Sokhadze, E. M., Baruth, J. M., Sears, L., Sokhadze, G. E., ElBaz, A. S., \& Casanova, M. F. (2012). Prefrontal neuromodulation using rTMS improves error monitoring and correction functions in autism. Applied Psychophysiology and Biofeedback, 37(2), 91-102. http://dx.doi.org/10.1007 /s10484-012-9182-5 
Sokhadze, E. M., El-Baz, A. S., Sears, L. L., Opris, I., \& Casanova, M. F. (2014). rTMS neuromodulation improves electrocortical functional measures of information processing and behavioral responses in autism. Frontiers in Systems Neuroscience, $\quad 8, \quad 134 . \quad$ http://dx.doi.org/10.3389 /fnsys.2014.00134

Wang, Y., Sokhadze, E., El-Baz, A., Sears, L., Tasman, A., \& Casanova, M. (2014). Prefrontal Neurofeedback Training Approaches in Autism. Proceedings of the 2014 ISNR Conference. NeuroRegulation, 1(3-4), 275-277. http://dx.doi.org/10.15540/nr.1.3-4.273

\section{The Clinical Use of Neurofeedback Treatment} for Major Depression Disorder

San-Yu Wang ${ }^{1}$, I-Mei Lin, PhD ${ }^{1}$, Yu-Ting Chen, MA ${ }^{1}$, Che-Cheng Chu, $M A^{1}$, Tze-Chun Tang, $P h D^{2}$, and YuChe Tsai, PhD ${ }^{1}$

${ }^{1}$ Kaohsiung Medical University, Kaohsiung, Taiwan

${ }^{2}$ Dr. Tang's Psychiatric Clinic and Mind Center, Kaohsiung, Taiwan

Background: Cortical hyperarousal was found in patients with major depressive disorder (MDD). The neurofeedback (NFB) is a kind of clinical intervention program for regulating brain activity and decreasing cortical hyperarousal. The purpose of this study was to explore the efficacy of NFB among patients with MDD.

Methods: Two-way mixed model and counterbalanced design was applied in this study. Twenty patients with MDD were randomly assigned to the NFB group and control group. There were nine MDD patients (mean age was $48.33 \pm 4.80$ years; two male and seven female) who participated in the NFB group that included 60 min weekly for 6 weeks. The protocol of NFB included sensor motor rhythm (SMR) uptrain and beta downtrain. The control group included 11 MDD patients (mean age was $46.27 \pm 13.21$ years; three male and eight female) that were treated as usual. There was no significant difference between the NFB group and control group in age $(t=0.44, p=.66)$ and gender $\left(X^{2}=0.07, p=.80\right)$. A 5-min resting baseline of electroencephalogram (EEG) was recorded at $\mathrm{Cz}$ by BrainAvatar (BrainMaster Technologies, Inc., Bedford, Ohio) before and after NFB training. The EEG power spectrum was analyzed by MATLAB ${ }^{\circledR}$ R2008a (MathWorks, Natick, Massachusetts) and EEG insight (Delorme \& Makeig, 2004) for beta power (13-32 Hz).

Results: There was a group * time interaction effect at pre-intervention and post-intervention between two groups, $F(1,18)=4.86, p<.05, \eta_{p}{ }^{2}=.025$. The post hoc comparison found lower beta power at post-intervention than that at pre-intervention in the NFB group $\left(17.87 \pm 5.78 \mu \mathrm{V}^{2}\right.$ and $\left.21.70 \pm 8.55 \mu \mathrm{V}^{2}\right)$; however, higher beta power at post-intervention than that at pre-intervention in the control group (13.01 \pm $7.88 \mu \mathrm{V}^{2}$ and $10.99 \pm 6.58 \mu \mathrm{V}^{2}$ ).

Conclusion: This study indicated that NFB can decrease beta power that may decrease cortical hyperarousal among patients with MDD.

\section{References}

Baehr, E., Rosenfeld, J. P., \& Baehr, R. (1997). The clinical use of an alpha asymmetry protocol in the neurofeedback treatment of depression: Two case studies. Journal of Neurotherapy, 2(3), 10-23. http://dx.doi.org/10.1300/J184v02n03_02

Baehr, E., Rosenfeld, P., Miller, L., \& Baehr, R. (2004). Premenstrual dysphoric disorder and changes in frontal alpha asymmetry. International Journal of Psychophysiology, 52(2), 159-167. http://dx.doi.org/10.1016/j.ijpsycho.2003.06.002

Delorme, A., \& Makeig, S. (2004). EEGLAB: an open source toolbox for analysis of single-trial EEG dynamics including independent component analysis. Journal of Neuroscience Methods, 134(1), 9-21. http://dx.doi.org/10.1016 /j.jneumeth.2003.10.009

Walker, J. E., Lawson, R., \& Kozlowski, G. (2007). Current status of QEEG and neurofeedback in the treatment of clinical depression. In J. R. Evans (Ed.), Handbook of Neurofeedback: Dynamics and Clinical Depression (pp. 341352). Binghampton, NY: Haworth Medical Press, Inc.

\section{Examining Correlations Between the TOVA} and the Evoke Continuous Performance Test Joshua Doidge and Andrea Reid-Chung, MA

ADD Centre and Biofeedback Institute of Toronto, Mississauga, Ontario, Canada

Children and adults with attention issues frequently show impaired performance on neuropsychological tests of attention. The Test of Variables of Attention, or TOVA, is a continuous performance test (CPT) that is widely used to assess whether a person has ADHD, as well as if a person would be a candidate for Neurofeedback training. The TOVA has also been used as a key measure in Neurofeedback studies that show how Neurofeedback can lead to improvements in attention (Gruzelier, Foks, Steffert, Chen, \& Ros, 2014; Lubar, Swartwood, Swartwood, \& O'Donnell, 1995; Thompson \& Thompson, 2005). The TOVA is a 21.6-min-long test, in which there are two black squares in a white box. The top square is the target to which clients must respond quickly and accurately, while inhibiting their response to the bottom square, which is not the target.

The TOVA has many measures of assessing attention, but there are four main measurements that are often examined as key variables: reaction time, reaction time variability, commission errors, and omission errors. Response time is the average time it takes a subject to respond correctly to a target. 
Response time variability is a measure of variability for accurate responses (Leark, Greenberg, Kindschi, Dupuy, \& Hughes, 2007). Commission errors occur when a subject fails to inhibit a response and presses the button to a non-target. Omission errors occur when a participant does not respond to a target and fails to press the button. Large deviations from the norm on any of these measures can indicate significant attention issues. While the TOVA test is considered a valid assessment of attention, would other tests that assess attention have similar results?

This study will investigate how the Evoke Neuroscience attention test correlates with the TOVA. The Evoke CPT is a 10-min test that can be used to assess attention issues. In the Evoke test, subjects must respond to a big blue circle but not push a button when a small blue circle pops up on the screen. During the Evoke CPT, the subject also undergoes a 19-channel EEG analysis, ERP analysis, and heart rate analysis, all of which allow clinicians to assess a client in multiple modalities simultaneously. Response time, response time variance, commission errors, and omission errors are presented in the Evoke assessment. This study will look at whether there are correlations between these four main measures in both the TOVA and the Evoke attention test by having subjects take both tests. We aim to recruit around 40 to 50 subjects for this study using both children and adults. Results with a smaller sample have been promising so far. There was a strong positive correlation between the percentage of omission errors in both tests $r(23)$ $=.740, p<.0005$. As for reaction time, there was a strong correlation between reaction time scores $r(23)$ $=.796, p<.0005$. Substantial correlations between the results would mean that if a client needed a multimodal assessment, or if a clinician or client were short on time, they could rely solely on the Evoke test to assess whether Neurofeedback training would be appropriate for a client.

\section{References}

Gruzelier, J. H., Foks, M., Steffert, T., Chen, M. J.-L., \& Ros, T. (2014). Beneficial outcome from EEG-neurofeedback on creative music performance, attention and well-being in school children. Biological Psychology, 95, 86-95. http://dx.doi.org/10.1016/j.biopsycho.2013.04.005

Leark, R. A., Greenberg, L. M., Kindschi, C. L., Dupuy, T. R., \& Hughes, S. J. (2007). Test of variables of attention continuous performance test. Los Alamitos, CA: The TOVA Company.

Lubar, J. F., Swartwood, M. O., Swartwood, J. N., \& O'Donnell, P. H. (1995). Evaluation of the effectiveness of EEG neurofeedback training for ADHD in a clinical setting as measured by changes in T.O.V.A. scores, behavioral ratings, and WISC-R performance. Biofeedback and Self-Regulation, 20(1), 83-99.
Thompson, L., \& Thompson, M. (2005). Neurofeedback intervention for adults with ADHD. Journal of Adult Development, 12(2), 123-130. http://dx.doi.org/10.1007 /s10804-005-7028-6

EEG Patterns Under Baseline, Erect, and Slouch: Body Postures Affect our Brain, Time to Memory Recall, and Correct Rate of Words Recall

Ying-Ju Chen ${ }^{1}$, Chia-I Ko ${ }^{1}$, Erik Peper, PhD ${ }^{2}$, and I-Mei Lin, $P h D^{1}$

${ }^{1}$ Kaohsiung Medical University, Kaohsiung, Taiwan

${ }^{2}$ San Francisco State University, San Francisco, California, USA

Background: Erect and slouch postures were related to energy level and the recall of positive and negative memories. Previous studies found that EEG patterns were related to different postures. The purpose of this study was to explore the EEG patterns under posture of baseline, erect, and slouch.

Methods: Twenty-eight healthy college students were recruited in this study (mean age was $20.64 \pm$ 1.06 years with 25 male and three female). ProComp Infiniti 6.0 (Thought Technology Ltd, Montreal, Quebec, Canada) was used to detect participants' EEG at $\mathrm{Cz}$ under posture of baseline, erect, and slouch with their eyes closed. EEG amplitudes of delta, theta, alpha, beta, low beta, and high beta were analyzed.

Results: This study found that there was significant higher slow wave amplitude (theta and alpha) at $\mathrm{Cz}$ under baseline than that under erect, $F(2,27)=$ $20.74, p<.001, \eta_{p}{ }^{2}=.43$, and slouch posture, $F(2$, $27)=7.08, p<.01, \eta_{p}^{2}=.21$; as well as higher delta amplitude at $\mathrm{Cz}$ under baseline than that under erect posture, $F(2,27)=5.38, p<.01, \eta_{p}^{2}=.17$. There was higher high beta at $\mathrm{Cz}$ under slouch than that under baseline and erect, $F(1.26,27)=11.16, p$ $<.01, \mathrm{n}_{\mathrm{p}}{ }^{2}=.29$.

Conclusion: This study found that higher slow wave EEG under baseline than that under slouch and erect might indicate that more relaxed state at baseline. The posture of slouch and erect may need more effort that was related to lower slow wave. The delta amplitude of erect was significantly lower than baseline and it indicated the possibility that erect posture might make people to be in a more alert situation. On the other hand, higher beta activity under slouch posture may relate to higher cortical arousal. 


\section{References}

Caldwell, J. A., Prazinko, B. F., \& Hall, K. K. (2000). The effects of body posture on resting electroencephalographic activity in sleep-deprived subjects. Clinical Neurophysiology, 111(3), 464-470. http://dx.doi.org/10.1016/S1388-2457(99)00289-8

Thibault, R. T., Lifshitz, M., Jones, J. M., \& Raz, A. (2014). Posture alters human resting-state. Cortex, 58, 199-205. http://dx.doi.org/10.1016/j.cortex.2014.06.014

Wilson, V. E., \& Peper, E. (2004). The effects of upright and slumped posture on the recall of postive and negative thoughts. Applied Psychophysiology and Biofeedback, 29(3), 189-195.

http://dx.doi.org/10.1023/B:APBI.0000039057.32963.34

The Relationships Between Heart Rate

Variability and Electroencephalography Under

Erect and Slouch Postures

Pei-Yun Lin ${ }^{1}$, Hsueh-Chen Lu' ${ }^{1}$ Erik Peper, PhD ${ }^{2}$, and IMei Lin, $P h D^{1}$

${ }^{1}$ Kaohsiung Medical University, Kaohsiung, Taiwan

${ }^{2}$ San Francisco State University, San Francisco, California, USA

Background: Body posture influences on our emotional states have been confirmed. Heart and brain interaction are related to our health and emotion regulation. Heart rate variability (HRV) is an index for heart autonomic activation, and electroencephalography (EEG) is an index of brain activity. This study was to explore the relationships between HRV and EEG under postures of baseline, erect, and slouch.

Methods: Twenty-eight healthy college students (age 20.64 \pm 1.06 years; female/male $=25: 3$ ) were invited to measure electrocardiography (ECG) and electroencephalography (EEG) by ProComp Infiniti 6.0 (Thought Technology Ltd, Quebec, Canada) under the following sequences: baseline, erect, and slouch posture. The interbeat intervals of ECG were transformed to HRV indices (SDNN, LF, HF, and LF/HF ratio) and the amplitude of delta, theta, alpha, and beta of EEG were analyzed. The change scores of HRV and EEG indices were calculated. The subjective emotion rating of happiness and depression were collected after erect and slouch posture.

Results: In erect posture, there were positive corrections between SDNN and amplitude of theta and alpha $(r=.42, p<.05 ; r=.43, p<.05)$; as well as LF and amplitude of theta and alpha $(r=.41, p$ $<.05 ; r=.38, p<.05)$. However, there was no relationship between $\mathrm{HF}$ and LF/HF ratio with EEG. In slouch posture, there was no correction between HRV and EEG indices. For the subjective emotion rating, there was higher happiness score at erect posture than that at slouch posture (64.50 and
59.62; $\mathrm{t}=2.38, \mathrm{p}<.05$ ); as well as higher of depression score in slouch posture than that in erect posture (24.97 and 19.14; $t=-2.71, p<.05)$.

Conclusion: In the erect posture, participants may tend to have higher happiness emotion than that at slouch posture; this situation may related to better vagal regulation and higher relaxation state. There were higher depression emotion in slouch posture than that at erect posture; however, there was no significant correction between HRV and EEG.

\section{References}

Chang, L.-J., Lin, J.-F., Lin, C.-F., Wu, K.-T., Wang, Y.-M., \& Kuo, C.-D. (2011). Effect of body position on bilateral EEG alterations and their relationship with autonomic nervous modulation in normal subjects. Neuroscience Letters, 490(2), 96-100. http://dx.doi.org/10.1016/j.neulet.2010.12.034

Sztajzel, J. (2004). Heart rate variability: A noninvasive electrocardiographic method to measure the autonomic nervous system. Swiss Medical Weekly, 134, 514-522.

Wilson, V. E., \& Peper, E. (2004). The effects of upright and slumped postures on the recall of positive and negative thoughts. Applied Psychophysiology and Biofeedback, 29(3), 189-195.

http://dx.doi.org/10.1023/B:APBI.0000039057.32963.34

\section{EEG Patterns Under Different Body Postures} and Emotion Stages

Hsin-Yi Tsai', Pin-Chun Chou', Erik Peper, PhD ${ }^{2}$, and I-Mei Lin, $P h D^{1}$

${ }^{1}$ Kaohsiung Medical University, Kaohsiung, Taiwan

${ }^{2}$ San Francisco State University, San Francisco, California, USA

Background: Previous study found that erect and slouch of body posture related to positive and negative emotion. Participants reported more positive emotion under erect posture; on the other hand, participants reported more negative emotion under slouch posture. This study explored EEG patterns under different body posture combined with positive/negative emotion recall.

Methods: Twenty-eight healthy college students were included in this study. The mean age was 20.64 years $(S D=1.06)$ with three male and 25 female. Lartin square design and counterbalance design was applied in this study. The $\mathrm{Cz}$ electroencephalogram (EEG) were recorded by ProComp Infiniti 6.0 (Thought Technology Ltd, Quebec, Canada) under the following sequences with eyes-closed baseline, erect with happiness recall, erect with depressive recall, slouch with happiness recall, and slouch with depressive recall. Each experimental stage was measured in $1 \mathrm{~min}$ to detect the EEG. The amplitudes of delta $(1-4 \mathrm{~Hz})$, 
theta $(4-8 \mathrm{~Hz})$, alpha $(8-12 \mathrm{~Hz})$, and beta $(12-32$ $\mathrm{Hz}$ ) were analyzed under five experimental stages.

Results: There were significant differences between five stages in delta, theta, and alpha amplitudes, the post hoc comparison found that there were higher delta, theta, and alpha at baseline than that at other stages $(F=5.37, p<0.01 ; F=6.34, p<0.001$; and $F=3.70, p<0.05$, respectively). On the other hand, there were significant differences between five stages in beta amplitude $(F=4.01, p<0.5)$, the post hoc comparison found that there was higher beta amplitude under slouch with happiness recall than that at erect with happiness recall; as well as higher beta amplitude under baseline than that at erect with depressive recall.

Conclusion: Higher slow wave activity may relate to relaxation stage under resting baseline than other postures. Slouch posture with happiness recall is a kind of inconsistent condition that may increase brain activity.

\section{References}

Peper, E., \& Lin, I.-M. (2012). Increase or decrease depression: How body postures influence your energy level. Biofeedback, 40(3), 125-130. http://dx.doi.org/10.5298/1081-5937-40.3.01

Wilson, V. E., \& Peper, E. (2004). The effects of upright and slumped postures on the recall of positive and negative thoughts. Applied Psychophysiology and Biofeedback, 29(3), 189-195.

http://dx.doi.org/10.1023/B:APBI.0000039057.32963.34

\section{The Relation Between Psychophysiological \\ Stress Profile and Integrated Visual and \\ Auditory Performance in Iranian National \\ Football Players (Under 14 Years) \\ Mohammadreza Ghasemian Moghadam ${ }^{1}$, Seyed Ata \\ Allah Aghilian, $P h D^{2}$, Masoud Nosratabadi, $P h D^{3}$, Mohammad Hossein Khaksar, $M A^{2}$, Freshte Azimpour ${ }^{3}$, and Mahnaz Ashegh Toosi, MA ${ }^{2}$ \\ ${ }^{1}$ Ferdowsi University of Mashhad, Mashhad, Iran \\ ${ }^{2}$ AREN Center, Mashhad, Khorasan Razavi, Iran \\ ${ }^{3}$ Parand, Tehran, Iran}

In the present study, the relation between Integrated Visual and Auditory (IVA) performance and Psychophysiological Stress Profile in Iranian national football players (under 14 years) was investigated. Based on previous works, psychophysiological stress profile is assessed during periods of rest and cognitive or perceptual and stressful tasks in order to understand the state of the autonomic nervous system and its responses to stressful stimuli. Stress assessment provides a holistic picture of what the mind/body of the athlete is doing at rest and during competitive tasks. This psychophysiological profile includes Heart Rate $(\mathrm{HR})$, Heart Rate Variability (HRV), Blood Volume Pulse (BVP), Skin Conductance (SC), Temperature $(\mathrm{T})$, Electromyography (EMG) and Respiration Rate $(\mathrm{RR})$ responses at rest and under stress. In order to evaluate integrated visual and auditory performance, the IVA test was used (Turner \& Sandford, 1995). The IVA is a test of visual and auditory attention and impulse control. In IVA test, visual and auditory in response control (RC) and attention scales were explored. The paired $t$ test showed that there are significant differences between rest and stress situation in Heart Rate, Heart Rate Variability, Skin Conductance, and Respiration Rate $(p<0.01)$. Based on results, in this group HR, RR, and SC responses are more sensitive to stress situation. At rest, there are correlations between HRV and attention ( $p=0.001, r=-0.8), \mathrm{HRV}$ and response control ( $p=0.001, r=-0.8)$ and also SC and response control $(p=0.044, r=-0.56)$. In addition, when changes in psychophysiological responses from resting to the stress situation were considered, visual response control was correlation with $\operatorname{RR}(p=$ $0.031, r=-0.6)$, SC $(p=0.03, r=-0.6)$ and BVP $(p$ $=0.001, r=-0.8)$. These results indicated that response control (error of commission, consistency, and stamina) especially in visual aspect is more related to stress responses.

\section{References}

Bezdjian, S., Baker, L. A., Lozano, D. I., \& Raine, A. (2009). Assessing inattention and impulsivity in children during the Go/NoGo task. British Journal of Developmental Psychology, 27(2), 365-383. http://dx.doi.org/10.1348/026151008X314919

Crocetti, A., Masaraki, S., Merati, S., Menotti, R., Forti, S., \& Aiello, G. (2010). Psychophysiological Stress Profile: A Protocol to Differentiate Normal vs. Pathological Subjects. Activitas Nervosa Superior Rediviva, 52(4), 241-245.

Dumont, R., Tamborra, A., \& Stone, B. (1995). Continuous Performance Tests: The TOVA, Conners CPT, and IVA. NASP Communiqué, 24(3), 22-24.

Eysenck, M. W., \& Calvo, M. G. (1992). Anxiety and performance: The processing efficiency theory. Cognition and Emotion, 6(6), 409-434. http://dx.doi.org/10.1080/02699939208409696

Turner, A., \& Sandford, J. A. (1995). A normative study of IVA: Integrated visual and auditory continuous performance test. Presented at the Annual Convention of the American Psychological Association, New York, NY. 
Intra-hemispheric Functional EEG Coherence

of Theta Rhythm in Successful and

Unsuccessful Golf Putting Performance in

Skilled Golfers

Chen Hoa, MA ${ }^{1}$, Tai Ting Chen, PhDc ${ }^{2}$, Kuo Pin Wang,

$M A^{2}$, and Tsung Min Hung, $P h D^{2}$

${ }^{1}$ Chinese Culture University, Taipei, Taiwan

${ }^{2}$ National Taiwan Normal University, Taipei, Taiwan

In the sport area when the athlete sustains attention for competition, there is a characteristic for achieving a superior athletic performance of precision sports, especially for golf players. When they are putting, that demands a high level of visuospatial motor control. Previous study has demonstrated that the frontal midline theta reflected higher levels of sustained attention and was associated with performance in golf putting (Kao, Huang, \& Hung, 2013) and basketball free throw (Chuang, Huang, \& Hung, 2013). In addition, the coherence analysis can provide more information about functional connectivity between different cortical areas in the brain. Therefore, the purpose of this study was to further investigate within the same hemisphere which cortical area connected with the other frontal area would play an important role in the successful and unsuccessful performance. We recruited 36 skilled golfers. All of the participants passed the eligibility of using the following exclusion criteria: history of psychiatric, neurologic, cardiovascular, or neuroendocrine diseases. All participants agreed with the experimental procedure and filled out the informed consent. The total of 40 golf putting at an artificial golf green; each block were 10 putts. The Coherence analysis 2 (performance: successful performance, unsuccessful performance) $\times 2$ (time: $\mathrm{T} 2=-2 \mathrm{~s} \sim-1 \mathrm{~s}, \mathrm{~T} 1=-1 \mathrm{~s} \sim 0 \mathrm{~s}$ ) $\times 8$ (coherence sites: F3-C3, F3-P3, F3-O1, F3-T3, F4-C4, F4-P4, F4-O2, F4-T4) ANOVA observed a significant interaction effect between performance and coherence sites, and we wanted to check this result. The post hoc simple main effect analysis indicated that the theta coherence of F3-P3 for successful performance in the brain was smaller than of the unsuccessful performances. The parietal cortex area has been associated with the somatosensory perception and integration of visual-spatial information (Schmidt \& Lee, 2011), the findings of present study suggests that sustained attention in integration of sensorymotor information was associated with golf putting performance.

\section{References}

Chuang, L.-Y., Huang, C.-J., \& Hung, T.-M. (2013). The differences in frontal midline theta power between successful and unsuccessful basketball free throws of elite basketball players. International Journal of Psychophysiology, 90(3), 321-328. http://dx.doi.org/10.1016/j.ijpsycho.2013.10.002

Kao, S. C., Huang, C. J., \& Hung, T. M. (2013). Frontal midline theta is a specific indicator of optimal attentional engagement during skilled putting performance. Journal of Sport and Exercise Psychology, 35(5), 470-478.

Schmidt, R. A., \& Lee, T. D. (2011). Motor Control and Learning: A Behavioral Emphasis (5th ed.). Champion, IL: Human Kinetics.

\section{The Comparison Between Sensorimotor}

Rhythm and Frontal Midline Theta During

Successful and Unsuccessful Putting

Performance in Skilled Golfers Under

Pressure

Tai Ting Chen, $P h D c^{1}$, Kuo Pin Wang, $M A^{1}$, Ming Yang

Cheng, PhDc ${ }^{2}$, and Tsung Min Hung, $P h D^{1}$

${ }^{1}$ National Taiwan Normal University, Taipei, Taiwan

${ }^{2}$ Cognitive Interaction Technology - Center of Excellence

(CITEC), Bielefeld University, Bielefeld, Germany

Introduction: Sensorimotor rhythm (SMR) is related with inhibition of irrelative sensorimotor information (Bowers, Saltuklaroglu, Harkrider, Wilson, \& Toner, 2014) and frontal midline theta $(F m \theta)$ is a good indicator of optimal attentional engagement (Kao, Huang, \& Hung, 2013). Both of them are attention systems, but SMR suppresses irrelative external sensorimotor information to enhance activity focused on expected sensory features, and $F m \theta$ reflects internal attention about action monitoring and cognitive control of sustained attention. Past studies have been shown that pressure could damage attention. However, it is still unclear whether SMR or $F m \theta$ would be damaged under pressure.

Purpose: The aim of this study was to examine and compare between SMR and Fm $\theta$ during successful and unsuccessful putting performance in pressure situations.

Methods: Thirty-one skilled golfers performed 40 putts at an individualized difficulty level of $50 \%$ putting success rate. Successful performances were those trials that made a hole, whereas unsuccessful performances were those that failed. In accordance with the International 10-20 system, electrode sites of brain waves were recorded on F3, F4, C3, C4, $\mathrm{P} 3, \mathrm{P} 4, \mathrm{~T} 3, \mathrm{~T} 4, \mathrm{O} 1$ and $\mathrm{O} 2$. Electrical reference was located on the left and right ear mastoids (A1, A2), and the ground electrode was located on FPz. Electrode impedance was kept below $5 \mathrm{k} \Omega$. In addition, band-pass filter was set at $1-30 \mathrm{~Hz}$. EEG data $2 \mathrm{~s}$ prior to the putting of the selected trials were segmented into two, and 1-s epochs for subsequent analysis. The sampling frequency was $500 \mathrm{~Hz}$. 
Results: Two-factor analysis of variance, 2 (performance: good, bad) $\times 2$ (time: T1, T2) demonstrated a significant effect of performance $X$ time interaction in Fm $\theta$ but not in SMR. Follow-up simple main effect analysis revealed: unsuccessful performance exhibited a larger decrease prior to putting. Moreover, the main effect of time of SMR and $\operatorname{Fm} \theta$ was significant.

Conclusions: Skilled golfers, no matter successful or unsuccessful putts, inhibited irrelative sensorimotor information and consumed less attentional resources prior to putting in pressure situations.

\section{References}

Bowers, A. L., Saltuklaroglu, T., Harkrider, A., Wilson, M., \& Toner, M. A. (2014). Dynamic modulation of shared sensory and motor cortical rhythms mediates speech and non-speech discrimination performance. Frontiers in Psychology, 5, 366. http://dx.doi.org/10.3389/fpsyg.2014.00366

Kao, S. C., Huang, C. J., \& Hung, T. M. (2013). Frontal midline theta is a specific indicator of optimal attentional engagement during skilled putting performance. Journal of Sport Exercise Psychology, 35(5), 470-478.

\section{A Randomized Controlled Trial Testing the Acute Impact of a Neurofeedback Device on Physiological Reactivity Carter Anne Baughman ${ }^{1}$, Omolola Fakunle ${ }^{1}$, and Sarah Wyckoff, $P h D^{2}$ \\ ${ }^{1}$ Centre College, Louisville, Kentucky, USA \\ ${ }^{2}$ Senselabs, Tempe, Arizona, USA}

Background: Stress regulation is an essential skill for a healthy functioning brain. As neuroscientists, we ask ourselves, "Is it possible for us to teach our brains to master this skill?" If so, can brief neurofeedback interventions be effective in significantly reducing levels of stress? These are questions that this study set out to answer. There is ample evidence that many stress-related disorders are characterized physiologically by imbalances or rigidity in the autonomic nervous system (Berntson et al., 1997; Berntson \& Cacioppo, 2004; Thayer, Friedman, \& Borkovec, 1996; Watkins, Grossman, Krishnan, \& Blumenthal, 1999). The efficacy of a course of neurofeedback therapy in the treatment of stress and anxiety has been established (Hammond, 2005; Moore, 2005; Sherlin, Gevirtz, Wyckoff, \& Muench, 2009; Sherlin, Muench, \& Wyckoff, 2010). The goal of this experiment is to assess the efficacy of one 20-min session of neurofeedback training on decreasing stress levels as compared to a placebo sham condition.
Methods: Participants in this study are individuals reporting mild, moderate, or severe levels of stress. The experiment begins and ends with the completion of the State-Trait Anxiety Inventory (STAI), so that the participant's perceived stress will be compared before and after the training session. An EEG brain assessment ( $\mathrm{Fz}, \mathrm{C} 3, \mathrm{Cz}, \mathrm{C} 4, \mathrm{Pz})$ of stress level using the Versus headset will also be compared before and after the training session. Throughout the experiment, brain activity is monitored and feedback to the participant is provided through the Versus brain training game "Neuroballoons." In the SHAM condition, the participants watch a prerecorded Neuroballoons training session, so they perceive themselves to be receiving feedback, but they are not. The brain training game uses operant conditioning to reinforce a participant for producing the desired, relaxed and calm yet alert, brain state by moving up levels and earning points. EEG data, physiological measures such as skin conductance, blood volume pulse, temperature, and EMG, and the STAI used as a selfreport measure, are collected, stored, and analyzed to better understand the efficacy of the Versus training game on stress. Arguably, the most important aspect of the study methodology is the pre and post brain assessments taken using the Versus headset. At the beginning and end of the experiment, the participant engages in a NeuroPerformance Assessment (NPA). This assessment contains both an eyes-closed and continuous performance task (CPT) portion. This assessment provides important information regarding the participant's brain activity both before and after the brief intervention.

Results: A repeated measures ANOVA will be used to analyze all continuous efficacy endpoints (prepost change in EEG, NPA variable, physiological measures, and STAI responses) with treatment group as the between-groups variable and measurement time point as the within-groups variable. Changes in these measures are useful in assessing the efficacy of the brain training game in modulating stress. Additional data analysis will include examining correlations between an individual's perceived stress, as indicated on the STAI, and their physiological stress demonstrated through bodily recordings.

Conclusion: This investigation is currently in process. The most current ANOVA and correlation data will be presented at the time of the conference. 


\section{References}

Berntson, G. G., Bigger, J. T., Eckberg, D. L., Grossman, P., Kaufmann, P. G., Malik, M., ... van der Molen, M. W. (1997). Heart rate variability: Origins, methods, and interpretive caveats. Psychophysiology, 34(6), 623-648.

Berntson, G. G., \& Cacioppo, J. T. (2004). Heart rate variability: Stress and psychiatric conditions. In M. Malik \& A. J. Camm (Eds.), Dynamic Electrocardiography (pp. 57-64). New York: Blackwell/Futura.

Hammond, D. C. (2005). Neurofeedback with anxiety and affective disorders. Child and Adolescent Psychiatric Clinics of North America, 14(1), 105-123. http://dx.doi.org/10.1016/j.chc.2004.07.008

Moore, N. C. (2005). The neurotherapy of anxiety disorders. Journal of Adult Development, 12(2), 147-154. http://dx.doi.org/10.1007/s10804-005-7031-y

Sherlin, L., Gevirtz, R., Wyckoff, S., \& Muench, F. (2009). Effects of respiratory sinus arrhythmia biofeedback versus passive biofeedback control. International Journal of Stress Management, 16(3), 233-248. http://dx.doi.org /10.1037/a0016047

Sherlin, L., Muench, F., \& Wyckoff, S. (2010). Respiratory sinus arrhythmia feedback in a stressed population exposed to a brief stressor demonstrated by quantitative EEG and sLORETA. Applied Psychophysiology and Biofeedback, 35(3), 219-228. http://dx.doi.org/10.1007/s10484-010-9132-z

Thayer, J. F., Friedman, B. H., \& Borkovec, T. D. (1996). Autonomic characteristics of generalized anxiety disorder and worry. Biological Psychiatry, 39(4), 255-266. http://dx.doi.org/10.1016/0006-3223(95)00136-0

Watkins, L. L., Grossman, P., Krishnan, R., \& Blumenthal, J. A (1999). Anxiety reduces baroreflex cardiac control in older adults with major depression. Psychosomatic Medicine, 61 334-340. http://dx.doi.org/10.1097/00006842-19990500000012

Results of the Neurofeedback Training in a Group of Adolescents in Conflict with the Law in Hermosillo Sonora Mexico, Combining a Protocol of One and Two Channels

Maria Gaemez, MD, and Teodoro Hernandez, MA

A Mas Centros, Hermosillo, Sonora, Mexico

Background: This study is a continuation of a previous study about the impact of the neurofeedback training in a group of juvenile offenders in the city of Hermosillo, Sonora, Mexico. In that study we reported the scores of the subscales of the WISC IV and the Stroop task in a protocol of Neurofeedback training (NFT) of one channel. In this study we reported the results in the subscales of the WISC IV, WAIS, and Stroop task in a group of juvenile offenders with a protocol of one and two channels combined.

Objective: The main objective of this study is to determine the effect of Neurofeedback training in the score of WISC IV, WAIS, and the Stroop task (Golden, 1978) in adolescents in conflict with the law housed in a Confinement Center after implementation of combining a protocol of one and two channels.

Methods: Participants: Eleven juvenile offenders, five participants were males and six female. The group ranged ages from 14 to 20 years, mean age of 16.73 years. All the participants are housed in a confinement center at the city of Hermosillo, Sonora. The training protocol selected consists of 39 sessions of $20 \mathrm{~min}$ each in order to increase beta (16-20 Hz), alpha $(8-13 \mathrm{~Hz})$ and coherence in beta and SMR $(13-15 \mathrm{~Hz})$. The training to increase alpha waves requires participants to relax with their eyes closed. The NFT was provided using the ProComp and BioGraph program (version 5.1.2; Thought Technology Ltd., Montreal, Canada). A sampling rate of $256 \mathrm{~Hz}$ with 2-s epochs was utilized. Skin impedance was less than $5 \mathrm{~K} \Omega$. NFT was based on a protocol proposed by Dr. Romano Micha.

Results: Pre- and post-training scores were compared. The Wilcoxon signed-rank test were performed to determine the changes in pre- and post-training scores from the WISC IV and WAIS; paired sample $T$-tests were performed to assess changes in pre- and post-training scores from the Stroop task. Significant differences were found at perceptual reasoning, and the processing speed indexes of WISC IV, and verbal and performance scores of the WAIS, as well as the scores of IQ in both. At the Stroop task, we found significant differences in the task of read, name color, name color in words, and the predicted score of name color in words task.

Conclusion: In spite of finding some statistical differences at the analysis data, it's very important to have a bigger sample of subjects that help to consolidate the impact of the NFT; in this study we have the opportunity of work with both sexes, male and female; however, we need more subjects for analysis and to research gender differences.

\section{References}

Golden, C. J. (2001). Stroop: Test de colores y palabras. (3rd Ed.). Madrid: TEA Ediciones.

Received: November 8, 2015

Accepted: November 8, 2015

Published: December 9, 2015 